\title{
Mobile e-learning course scenario model on PDA
}

\author{
J. Schreurs ${ }^{1}$ \\ ${ }^{1}$ Hassselt University, Diepenbeek, Belgium
}

\begin{abstract}
Web-based services mobile learning can enable collaborative learning and access to different information sources in actual problem-solving situations. The PDA's are now designed to be smaller and sleeker and are popular due to its portability and facility for wireless connection. Handheld computers can become an integral part of the learning activities. But due to the very small screen, limited memory capacity and a large diversity of mobile devices, a handheld seems to obstruct a good learning experience. Handling of learning materials is a challenge. Experimentation with learning path scenarios and based on the e-mindmap concept is set forward here.
\end{abstract}

Index Terms - A Mobile learning, PDA, mobile internet, Learning objects, scenario model, e-mindmap

\section{A PDA (PERSONAL DIGITAL ASSISTANT)}

Mobile learning means the provision of education and training materials and courses on wireless devices: portable computers, PDA's (personal digital wireless devices), and mobile telephones. We concentrate here on the PDA, as a handheld computer.

Together with web-based services mobile learning can enable collaborative learning and access to different information sources in actual problem-solving situations

The needs of being able to access information anytime and anywhere makes personal digital assistants (PDA's) more popular due to its portability and facility for wireless connection. The PDA's are now designed to be smaller and sleeker.

With a PDA, a teacher, student or administrator can do amazing things: take notes, calculate, sketch ideas, collect data, access resources, manage activities and, with the right hardware, even access the Internet wirelessly. Handheld computers can offer unique benefits to students and teachers. Students can have a personal, portable device ready-at-hand for individual or collaborative learning activities, wherever they go. Students can use handheld computers to collect data in the field, to learn vocabulary words while waiting to be picked up after soccer practice, or to self-quiz during a long car ride.

\section{A PDA FOR LEARNING: A CHALLENGE}

\section{A. PDA functions}

The PDA's are advancing to a more powerful device and equipped with increasing numbers of features. Word processors, personal schedulers, e-mailing, language programming and other traditional desktop applications are increasingly available on this platform.
On the technological side we have seen some developments that can give a boost to mobile learning. On the one hand there is the upcoming usage of GPRS and UMTS, this makes it possible to send/receive data at a higher speed. On the other hand there is a large increase of functionality and usage of mobile devices. One of the last developments is the support of TCP/IP, http-protocols within WAP2.0. This makes mobile internet applications possible and gives access to general web page formats.

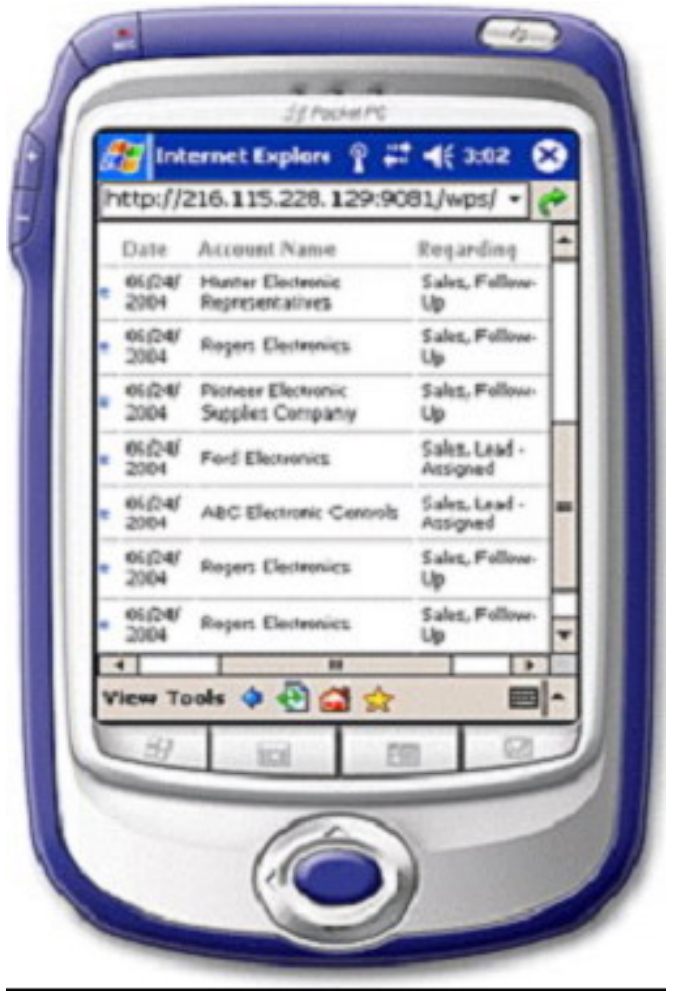

Figure 1. PDA showing a list of contacts

\section{B. Handheld computers integrated in the flow of learning activities}

Handheld computers can become an integral part of the learning activities by integrating it, the software and the peripherals, into them. They seem to be more easily integrated with the flow of learning activities than a desktop. The challenge lies in using mobile technologies well, both as an enhancer in the classroom and to bridge arenas that are usually referred to as separate, such as workplace and free time.

First the handheld will not replace the desktop in learning. A mix of facilities can be used where handheld can be useful enhancing rather than disruptive technology and can be an integral part of workplace and life. So can a 
desktop be used for online browsing, with the info that the learners found relevant downloaded to their handheld using an offline browser. And as stated by J.Liebowitz(2007): "In the affective domain, students asserted highly positive attitudes towards the use of wireless appliances.”

\section{Small screens seems to obstruct a good learning experience}

Nevertheless they have very small screens, limited memory capacity and the large diversity of mobile devices obstruct a good learning experience. (figure 1)

The typical characteristics of easy handling of a handheld because of its small size, seem to obstruct a good learning experience. Following L.Uden (2007): “The environmental constraints of mobile devices, such as limited processing power and memory, affect not only the functional aspects of these devices but also the user interface.”

\section{Handling of learning materials: a challenge}

In using handheld in learning, the learners are limited and have to be selective in what they chose to download and establish a set of criteria for making their choices. Also the mobile use of a web-based learning management system is not possible because of its heavyweight multimedia content. We will have to get around the technical restrictions so we can create a good learning experience. First the usage of video, audio, clear interfaces and divided courses must contribute to a solution. Second the learning materials must answer to specific conditions. The learning content will be defined as learning objects and managed in a central database. Based on these objects more e-learning course scenario models are built, that can be used via handheld or via desktop.. Dependent on the used appliance by the learner, being a desktop or an PDA, the e-learning course will be presented in another presentation scenario

\section{E-LEARNING COURSE MODULE BUILT FOLLOWING \\ A SCENARIO MODEL}

We have to adapt the content to the needs of the mobile user. Because he has a very fragmentized time schedule, we will have to be sure that the learning object are not to long. Dividing the knowledge in smaller modules offers a solution. We decompose the learning content into a set of smaller content components or blocks. Those blocks contain a small part of learning content (block-full text doc). Than in the instructional design phase we define a scenario model and add some additional information and some interactive and animation components (abstracts or short text docs, figures, videos, Q\&A, keywords, html links, audio fragment,...) to the content layer and define the presentation layer. We can create different compositions or scenario models of the same set of blocks to present the learning content to the learner as an elearning course module.

\section{A. Decomposition of the learning content into Learning Content components.}

The learning content has been decomposed into a set of smaller content components or blocks. The blocks contain a small part of learning content (block-full text doc) and can contain some additional information and some interactive and animation documents. (Abstracts or short text docs, figures, videos, Q\&A, keywords, html links, audio fragment,...). Afterwards a certain composition of blocks will be designed, linked with the chosen e-learning concept fulfilling the requirements corresponding to the personal learning style of the learner. The architecture of the block itself is important. Which additional supporting, interactive and animating elements will be linked with the block content and how will it be presented to the learner, or which scenario model will be chosen?

\section{B. A scenario model}

In instructional design a scenario model has to be defined. A composition of blocks will be defined and the architecture of the blocks will be designed. All additional elements are parts of the presentation layer. The learning content included in the block (being the full text document) will be supplemented with other supporting, interactive and animating elements, being all parts of the presentation layer.

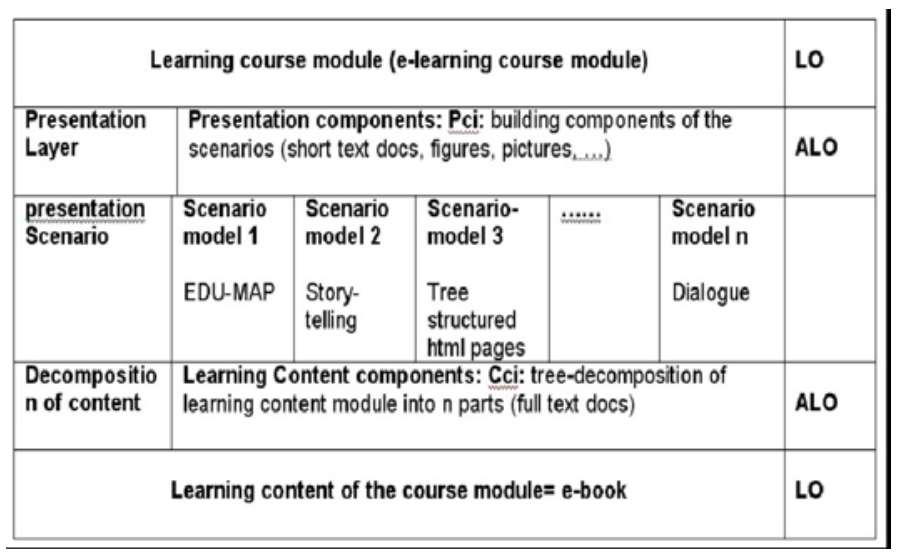

Figure 2. Scenario models

The presentation layer is composed of presentation components put above the same original content components. Some of the presentation components are content related and others are more design/format related. An example of content related presentation component: someone tells the story of a best practice. An example of design related presentation component: a live picture of the storyteller is shown on the screen The presentation components are belonging to one or more scenarios models. The e-learning course module and the learning content itself are learning objects (LO). The content- and the presentation- components are atomic learning objects (ALO).

\section{Presentation of learning content as a scenario model}

We can create different compositions or scenario models of the set of blocks to present the learning content to the learner as an e-learning course module. The blocks are the building blocks and linked with them are the basic atomic learning objects.

Not only the blocks are presented in a different composition, the blocks themselves differ in architecture by using the atomic learning objects on a different way. 


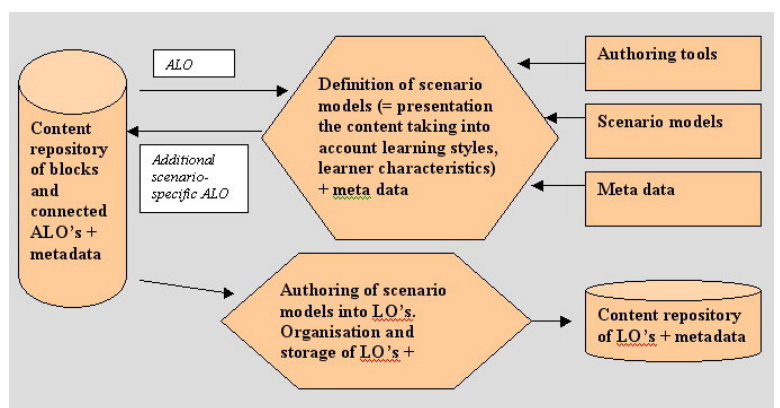

Figure 3. Process of LO creation

More and different scenarios can be built upon the same decomposition of the learning content. Dependent on the type of scenario model, a set of presentation components or atomic learning objects, will be defined and are brought together into a scenario model design. The learning objects (LO) structure corresponding to a scenario model, has to be defined and the corresponding relational data model has to be customised. Some of the presentation components are common to different scenarios and can be re-used. Others are very specific for the scenario to which they belong. The structure of the Learning Object database has been set up to contain all kind of presentation components required to build e-learning courses following several scenario models.

Following the traditional "web-pages e-learning "concept, we can built a tree structured html scenario model. We can bring together the full text docs of all blocks and put them in the pre-defined tree structure. On this time the block, being a html page must be designed using the presentation of the full text and the other atomic elements, being figures, hypertext links, Q\&A, keywords, audio fragments, ... can be linked with it following the design of the scenario model.

Following the e-MINDMAP concept, we can build an EDUMAP scenario model.

We can organize the blocks in a graphical way in units and in EDUMAPS. Relevant atomic objects are the full text, the short text, figures, video's, ....

The datamodel is very similar to this of the previous scenario model.

\section{D. e-MINDMAP scenario}

The e-EDUMAP is so telling the story in a graphical way and in a sequence of steps. The blocks are composed of some atomic learning-elements, being the short text or audio document, the full text, some additional text or graphical presentations, or pictures, some questions and answers, some tests, some mouse-over animations, .... .

The most challenging characteristic of the e-MINDMAP is the visual way of structuring the content. Animation can be built in too. The learner will be supported by this way to understand and to print in memory the structured content with the underlying relations between the content blocks. An example of a course module following the eMINDMAP concept:

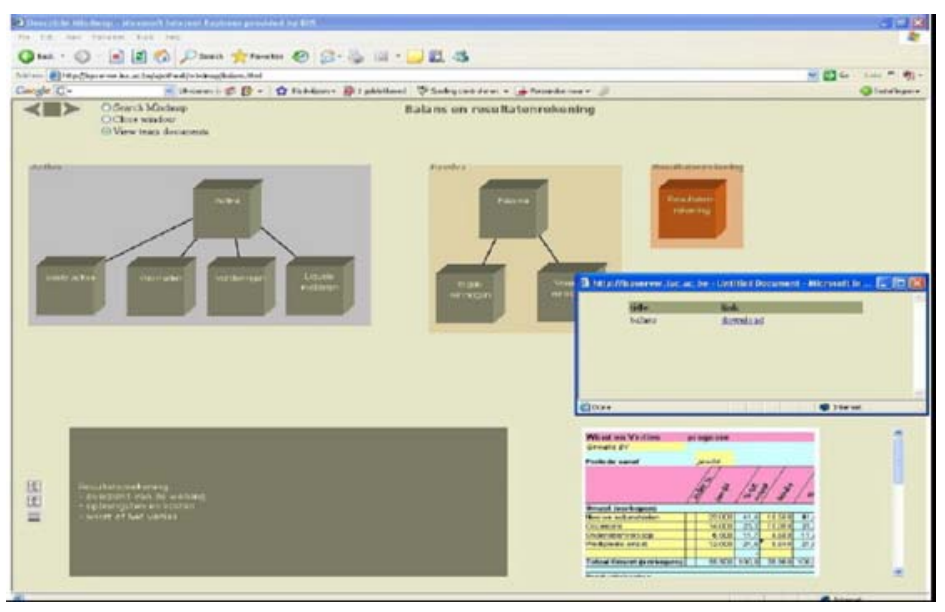

Figure 4. e-Mindmap

\section{A LEARNING PATH SCENARIO APPLIED FOR LEARNING ON PDA}

The learning content will be defined as learning objects being a composition of information (atomic ) objects, following a presentation scenario

Dependent on the used appliance by the learner, being a desktop, a portable or a PDA, the e-learning course will be presented in another presentation scenario, using the same ALO's

\section{A. Example: a virtual walk through history}

Historical information about the region between Aachen (Germany) and Eupen (Belgium) has been made available for interested people. In this region one can visit some historical places known for the escape of the Jewish people to Belgium. Other facts relate to the smuggling of coffee from German to belgium. A walk in the border region is advised to become a real feeling of these historical facts. People can learn about the historical elements of a region. The historical info of a region has to be delivered via internet. We implemented a virtual walk in that region. The application has been built so that deep learning can take place on PC and the touristic info-level can take place on PDA. We put the minimum set of information on the PDA. During the walk the information linked with some physical places can be accessed. On the same way can the application been used as a virtual walk on PC. As a scenario we have chosen for showing the map and we linked the information with the historical points indicated on it.

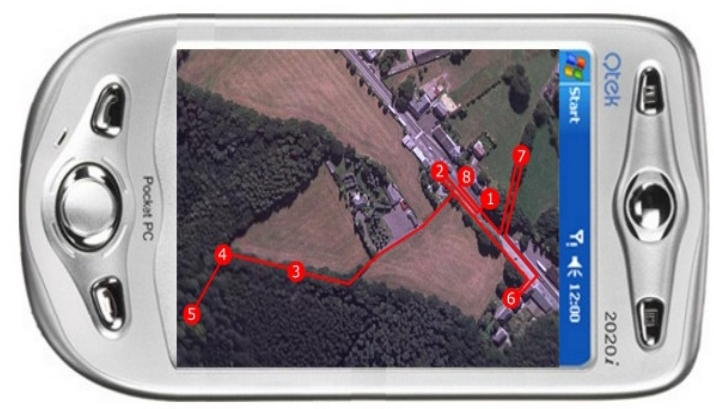

Figure 5. Information path 
The information is delivered following the mindmap principle, but limited to one unit of information at a time. We have limited the written info and replaced it with spoken info and delivered a lot of pictures. The PDA is a real guide during the walk, showing pictures and telling us some historical facts of the places when we are physically there.

\section{CASE KBC}

The case study used is based on the design of a mobile learning environment for students to construct a knowledge delivery and e-learning solution for the Belgium bank KBC. Students were working as a team and each student was given a handheld PDA to use in this project. $\mathrm{KBC}$ is a Belgium Bank being also active in Central and East Europe. A KBC business analyst working on a pre-study in Poland needs access to knowledge available on a central computer in Belgium. First the business model as well the ICT architecture for the KBC Central Europe application "Credits" have been developed. It is required that the analyst can querry the information on distance and on a flexible way via mobile internet and by using a PDA. The same knowledge is structured as supporting information in a framework WVF and is structured in learning objects to be used in learning about the business analysing process.
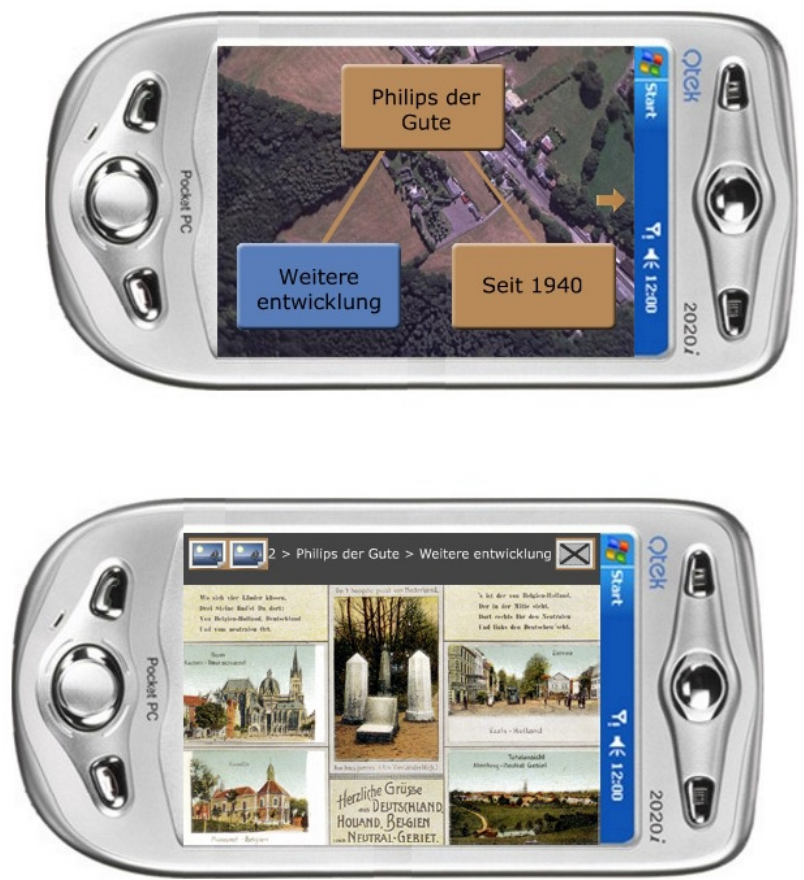

Figure 6. Limited mindmap and pictures

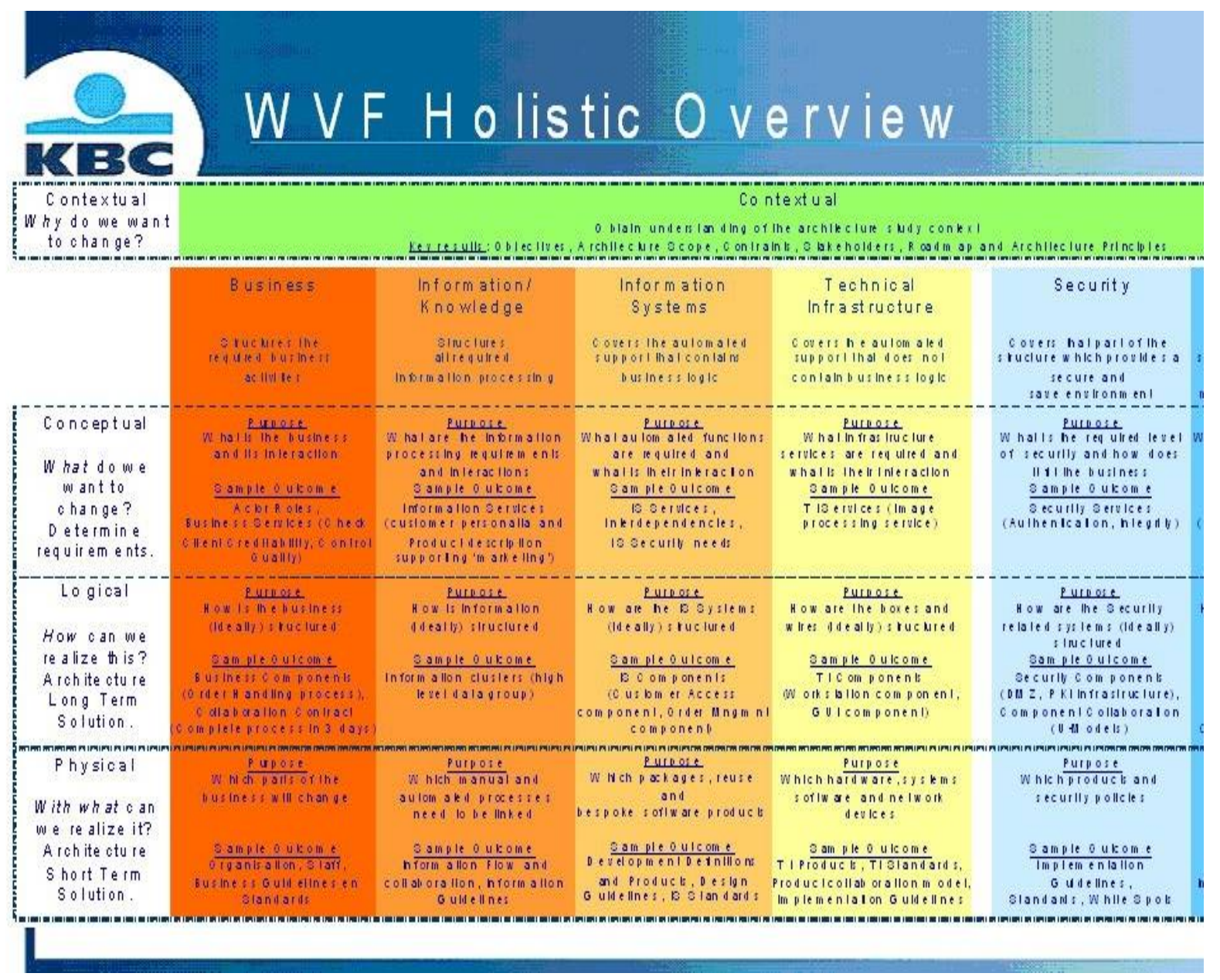

Figure 7. The WVF schema 
In a student project, the students had to develop an elearning course based on mobile internet and to open the access to information behind the WVF scheme via PDA. They structured the (learning) content in learning objects and they tried to find the best (course ) presentation layer for use on PDA. and create a PDA course re-using the same learning objects. The best scenario has been found to present the querried information and to learn the fundamentals of business analysis.

The WVF scheme is rather complex and too big to be shown on PDA. The overview of the cells without any additional explanation is shown. The information itself can be found by clicking on the related linkages.
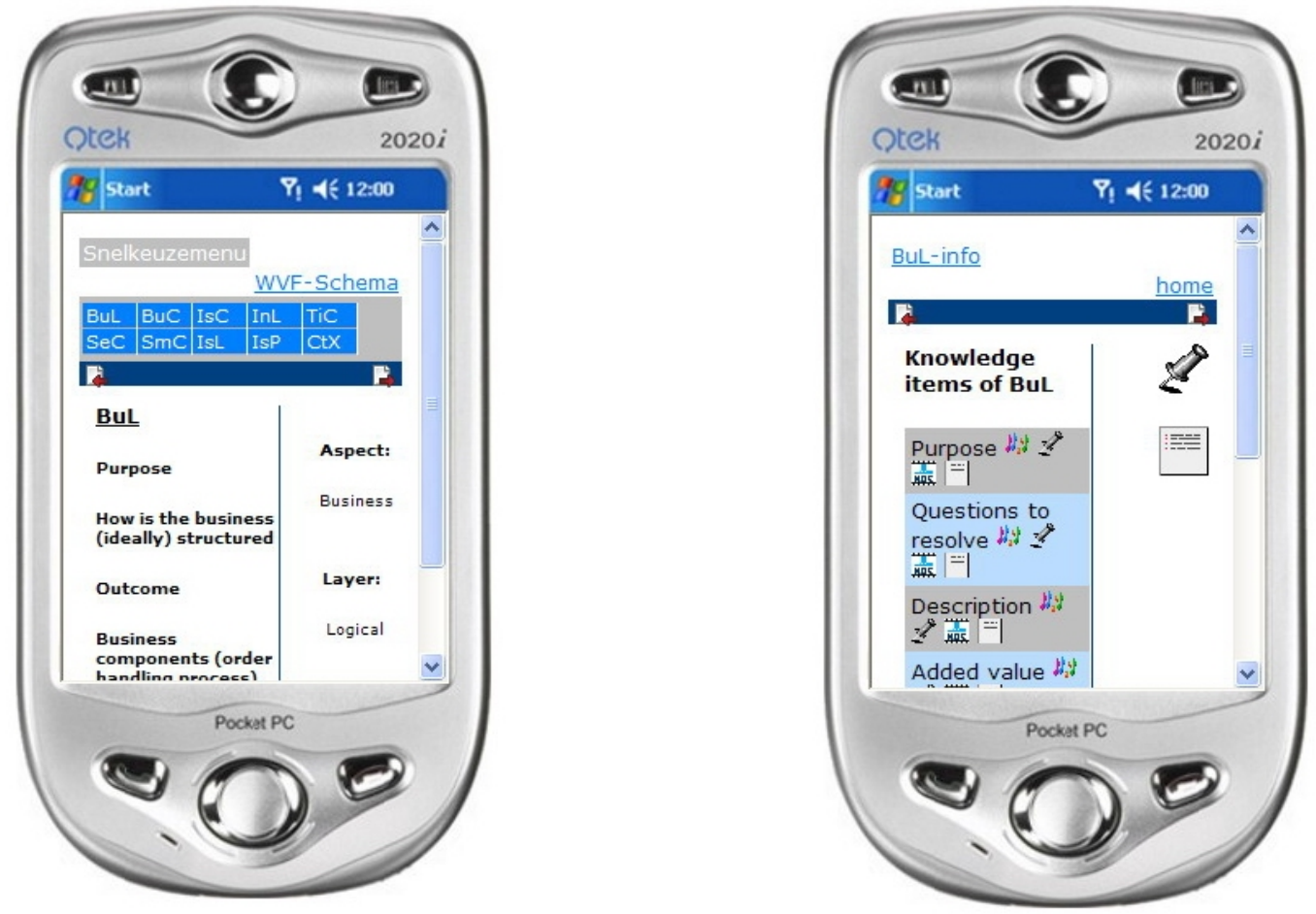

Figure 8. Overview WVF structure on PDA and the decomposition of the content in content topics

The use of text has been limited and replaced by voice output. The information behind each cell has been structured in some information categories : purpose, questions to resolve, .... And for each of them we have split again the content into information objects or the ALO's: a short text document, a video fragment, a figure, ....

We managed it very well to bring the content in a fragmented and though integrated way. The only thing we can not solve is the presentation of graphs. The only solution for them is to present a graph in a very small scale and than enlarge a selected part, having at the same time the possibility of moving the selection. The same principle is used in showing maps on the PC screen.

\section{CONCLUSION}

Handheld computers have become an integral part of the learning activities. But due to the very small screen, limited memory capacity and a large diversity of mobile devices, a handheld seems to obstruct a good learning experience. Experimentation with learning path scenarios and based on the e-mindmap concept is set forward here. A learning path scenario has been applied in the application of the delivery of historic information about a region. It is based on the e-mindmap concept that has been limited this time to one unit. In the case $\mathrm{KBC}$ the challenge is to find a solution or a scenario model for the complex frame. The risk is high that the overview is being lost. We managed it very well to bring the content in a fragmented and though integrated way. The only thing we can not solve is the presentation of graphs. The only solution for them is to present a graph in a very small scale and than enlarge a selected part, having at the same time the possibility of moving the selection. The same principle is used in showing maps on the PC screen. 


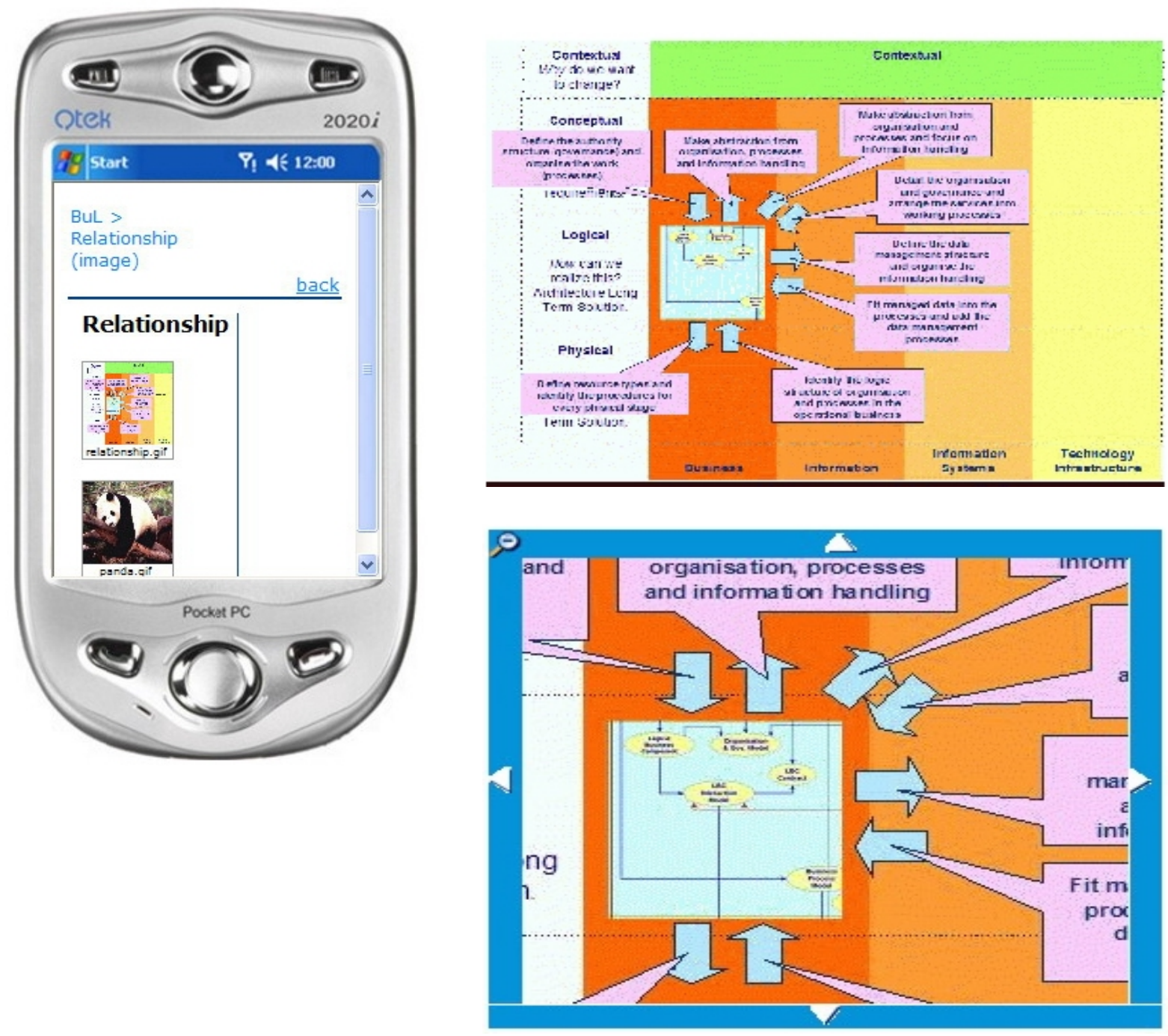

Figure 9. Presentation of a graph on PDA

\section{REFERENCES}

[1] Jeanne Schreurs: m-learning using PDA's and our supporting LOMS." International Journal ofcomputing and information sciences (IJCIS); Vol 4 No 2 (2006); page 72; ISSN 1708-0460.

[2] Jeanne Schreurs, Maarten Steegmans, Rachel Moreau, Johan Broun: M-learning LOMS » inproceedings of G GAME'ON'2004 Conference ; Gent 25-27 nov 2004

[3] Jeanne Schreurs, Rachel Moreau: Presentation of learning content as an e-MINDMAP andputting together more learning styles, to improve the e-learning adoption. Proceedings of KAM'2005 conference Lubanski May 12-14; ISSN 0324-8445; pages 246256.

[4] Jeanne Schreurs, Rachel Moreau: "Converting learning content to Learning Objects (LO) andatomic LO's" in Proceedings of the virtual conference: IADIS Virtual Multi Conference on Computer Science and Information Systems (MCCSIS 2006) 15-189 May 2006. ISBN 972-8924-14-3
[5] Jay Liebowitz: Developing knowledge and learning strategies in mobile organizations; Int. J. Mobile Learning and Organization. Vol 1, No1, 2007; 5-14.

[6] Lorna Uden: Activity theory for designing mobile learning; Int. J. Mobile Learning and organization, Vol 1, No1; 81-102.

\section{AUTHORS}

J. Schreurs is Professor at the Faculteit Toegepaste Economische Wetenschappen,

Universiteit Hasselt, Campus Diepenbeek, Agoralaan gebouw D, 3590 Diepenbeek, Belgium.

(e-mail: Jeanne.Schreurs@uhasselt.be).

Manuscript received juli 2007

Published as submitted by the author(s). 